\title{
Extended emission around GPS radio sources
}

\author{
C. Stanghellini ${ }^{1}$, C. P. O’ Dea $^{2}$, D. Dallacasa ${ }^{1,4}$, P. Cassaro ${ }^{6}$, S. A. Baum ${ }^{3}$, R. Fanti ${ }^{1,5}$, and C. Fanti ${ }^{1,5}$ \\ 1 Istituto di Radioastronomia - INAF, Via Gobetti 101, 40129 Bologna, Italy \\ e-mail: cstan@ira.cnr.it \\ 2 Department of Physics, Rochester Institute of Technology, 54 Lomb Memorial Drive, Rochester, NY 14623, USA \\ 3 Center for Imaging Science, Rochester Institute of Technology, 84 Lomb Memorial Drive, Rochester, NY 14623, USA \\ 4 Dipartimento di Astronomia, Università degli Studi, via Ranzani 1, 40127 Bologna, Italy \\ 5 Dipartimento di Fisica, Università degli Studi, via Irnerio 46, 40126 Bologna, Italy \\ ${ }^{6}$ Istituto di Radioastronomia - INAF, CP 141, 96017 Noto SR, Italy
}

Received 21 October 2004 / Accepted 22 June 2005

\section{ABSTRACT}

Extended radio emission detected around a sample of GHz Peaked Spectrum (GPS) radio sources is discussed. Evidence for extended emission which is related to the GPS source is found in 6 objects out of 33. Three objects are associated with quasars with core-jet pc-scale morphology, and three are identified with galaxies with symmetric (CSO) radio morphology. We conclude that the core-jet GPS quasars are likely to be beamed objects with a continuous supply of energy from the core to the kpc scale. It is also possible that low surface brightness extended radio emission is present in other GPS quasars but the emission is below our detection limit due to the high redshifts of the objects. On the other hand, the $\mathrm{CSO} /$ galaxies with extended large scale emission may be rejuvenated sources where the extended emission is the relic of previous activity. In general, the presence of large scale emission associated with GPS galaxies is uncommon, suggesting that in the context of the recurrent activity model, the time scale between subsequent bursts is in general longer than the radiative lifetime of the radio emission from the earlier activity $\left(\sim 10^{8} \mathrm{yr}\right)$.

Key words. galaxies: active - quasars: general - radio continuum: galaxies

\section{Introduction}

The GHz-peaked-spectrum (GPS) radio sources are powerful but compact $(<1 \mathrm{kpc})$ radio sources whose spectra are generally simple and convex with a peak near $1 \mathrm{GHz}$. The GPS sources make up $\sim 10 \%$ of the bright extragalactic source population at $\mathrm{cm}$ wavelengths. Common characteristics of the bright GPS radio sources are: high radio luminosity, low fractional polarization, and, apparently, low variability. The optical identifications include both quasars and galaxies. The galaxies tend to be $L_{*}$ or brighter and at redshifts $0.1 \lesssim z \lesssim 1$ (O'Dea et al. 1996) while quasars are often found at very large redshifts $1 \lesssim z \lesssim 4$. A review of GPS radio sources has been presented by O'Dea (1998).

Two basic models have been proposed to explain the subgalactic sizes of the GPS and the slightly larger CSS (Compact Steep Spectrum) radio sources.

(1) In the frustration model (e.g., van Breugel et al. 1984; De Young 1993; Carvalho 1994, 1998) the radio emitting plasma is confined (for the lifetime of the radio emission) to a region within the host galaxy by an external medium which is dense and/or clumpy enough to prevent the expansion of the radio source.
(2) The youth model (Phillips \& Mutel 1982; Fanti et al. 1995; Readhead et al. 1996; O’Dea \& Baum 1997; Alexander 2000; Snellen et al. 2000, 2003) suggests that GPS sources with double or triple morphology are small because they are young and that during the course of their life they will propagate outwards while decreasing in radio luminosity by about one order of magnitude for a factor 100 in size.

Both the the "youth" and the "frustration" scenarios are motivated by the morphological similarity between the small GPS/CSS galaxies and the extended radio galaxies.

The detection of arc-second scale faint extended emission around 0108+388 (Baum et al. 1990) and other GPS radio sources (Stanghellini et al. 1990), motivated the suggestion, in the framework of the youth scenario, that nuclear activity is recurrent in these sources. In this hypothesis, we see the relic of a previous epoch of activity as faint diffuse emission surrounding the current young nuclear source.

In this paper we present deep VLA L band images of the 1 Jy complete sample of bright GPS radio sources (Table 1) defined by Stanghellini et al. (1998). We search for extended (tens/hundreds $\mathrm{kpc}$ ) radio emission which could be associated with the GPS source. We discuss the characteristics of the positive detections, the likelihood of their association with the 
Table 1. Radio emission data of the sources in the three epochs of the observations $(a=22$ Aug. 1998; $b=10$ Sep. 1998; $c=21$ Sep. 1998). Column [1], IAU name; Col. [2] optical id.; Col. [3] redshift (values in parentheses are photometric redshifts, using the GPS galaxy Hubble diagram); Cols. [4]-[7] flux density of the arc second core and any additional radio emission at 1.36 and $1.66 \mathrm{GHz}$ (values in parentheses indicate that in our judgment, the radio emission likely comes from an unrelated object). Flux density errors for compact strong components are dominated by the calibration uncertainties estimated around 3\%, while for extended weak components the flux density errors are dominated by the limits of the self-cal/imaging process in the presence of a strong source nearby, and the flux densities given can be considered only rough estimates. * It accounts for the region South of the arcsecond core only.

\begin{tabular}{lcc|rr|rr}
\hline \hline & & \multicolumn{2}{|c}{$1.36 \mathrm{GHz}$} & \multicolumn{2}{c}{$1.66 \mathrm{GHz}$} \\
\hline Name & id & $z$ & Peak & Extended & Peak & Extended \\
& & & mJy & mJy & mJy & mJy \\
\hline B0019-000a & G & 0.305 & 2932 & - & 2652 & - \\
B0108+388ac & G & 0.669 & 414 & 9.3 & 595 & 6.0 \\
B0237-233a & Q & 2.223 & 6282 & $(62.0)$ & 5950 & $(51.5)$ \\
B0248+430a & Q & 1.310 & 1197 & $(26.2)$ & 1281 & $(25.1)$ \\
B0316+162a & G & $(1.57)$ & 7820 & $(25.0)$ & 7167 & $(21.9)$ \\
B0428+205a & G & 0.219 & 3720 & - & 3665 & - \\
B0457+024a & Q & 2.384 & 2242 & - & 2417 & - \\
B0457+024c & Q & 2.384 & 2248 & - & 2232 & - \\
B0500+019a & G & 0.583 & 2144 & $(42.1)$ & 2341 & $(30.5)$ \\
B0710+439a & G & 0.518 & 1953 & - & & \\
B0738+313a & Q & 0.631 & 2036 & 86 & 2461 & 57 \\
B0742+103a & Q & 2.624 & 3308 & - & 3795 & - \\
B0743-006a & Q & 0.994 & 690 & $(25.2)$ & 841 & $(17.9)$ \\
B0941-080ab & G & 0.228 & 2725 & 31.1 & 2401 & 24.4 \\
B1031+567a & G & 0.460 & 1791 & - & 1741 & - \\
B1117+146a & G & 0.362 & 2445 & - & 2153 & - \\
B1127-145b & Q & 1.187 & 5448 & 96.2 & 5371 & 75.4 \\
B1143-245b & Q & 1.940 & 1557 & - & 1676 & - \\
B1245-197b & Q & 1.273 & 5322 & $(54.6)$ & 4708 & $(45.4)$ \\
B1323+321a & G & 0.369 & 4855 & - & 4339 & - \\
B1345+125ac & G & 0.122 & 5262 & 35.4 & 4734 & 32.1 \\
B1358+624c & G & 0.431 & 4357 & - & 3860 & - \\
B1404+286a & G & 0.077 & 792 & $(7.3)$ & 1070 & $(5.8)$ \\
B1442+101a & Q & 3.522 & 2432 & - & 2220 & - \\
B1518+047a & Q & 1.296 & 3963 & $(243)$ & 3457 & $(211)$ \\
B1600+335a & G & $(1.10)$ & 2914 & - & 2834 & - \\
B1607+268a & G & 0.473 & 4875 & - & 4387 & - \\
B2008-068a & G & $(0.70)$ & 2576 & $(55.4)$ & 2523 & $(58.3)$ \\
B2126-158a & Q & 3.270 & 562 & - & 684 & - \\
B2128+048a & G & 0.990 & 3970 & $(19.2)$ & 3747 & $(18.5)$ \\
B2134+004a & Q & 1.932 & 3412 & $11.0 *$ & 4608 & $7.1 *$ \\
B2210+016a & G & $(0.68)$ & 2823 & - & 2522 & - \\
B2342+821a & Q & 0.735 & 3797 & - & 3286 & - \\
B2352+495a & G & 0.237 & 2532 & - & 2390 & - \\
\hline & & & & &
\end{tabular}

GPS source, and the implications for models of GPS source growth/evolution.

$H_{0}=100 \mathrm{~km} \mathrm{~s}^{-1} \mathrm{Mpc}^{-1}$, and $\mathrm{q}_{0}=0.5$ have been used throughout this paper.

\section{The observations}

The observations presented here were carried out with the VLA in B configuration in 3 separate sessions on 22 August, 10 September, and 21 September 1998, at $1335 \mathrm{MHz}$ and
$1665 \mathrm{MHz}$ with a bandwidth of 50 and $25 \mathrm{MHz}$ respectively. All the sources of the $1 \mathrm{Jy}$ complete sample were observed. Each source has been observed for a total time ranging from 5 to $20 \mathrm{~min}$. The typical rms noise in the final images is in the range $0.1-0.5 \mathrm{mJy}$, and the angular resolution is about 5 arcsec.

We also used VLBI images for a comparison with the VLA data when it was necessary for a better understanding of the source structure. We used VLBI data 


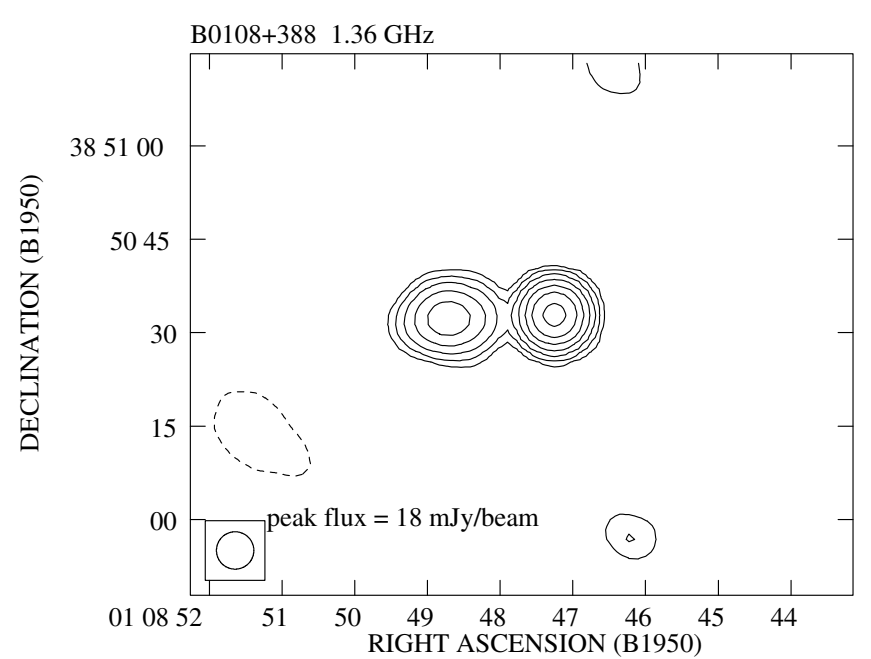

Fig. 1. $0108+388$ at $1.36 \mathrm{GHz}$. $0.4 \mathrm{Jy}$ has been subtracted at the core position to improve the detection of extended emission close to the compact component.

from Stanghellini et al. (1997, 2001) and the Radio Reference Frame Image Database (RRFID) whose calibrated UV data sets were kindly provided by Alan Fey (http://rorf.usno.navy.mil/rrfid.shtml). The latter were imaged by us with appropriate weighting and/or tapering to enhance either the resolution or the sensitivity to extended emission on the pc scale (see also Fey et al. 1996, for the original images).

We also examined the images from the NRAO VLA Sky Survey (NVSS) at $1.4 \mathrm{GHz}$ (Condon et al. 1998) to look for indications of extended emission on the arcmin scale (the resolution of the images from the NVSS is about 45 arcsec).

\section{Results}

We found several objects with diffuse extended emission or a secondary component close to the dominant GPS radio source. Here we discuss these sources in detail. In Table 1 and Figs. 1-17 we summarize the results of the VLA observations. In sources where extended emission was seen, we superimposed the radio image on an optical image either from the POSS2 or, when available, from our Nordic Optical Telescope observations (Stanghellini et al. 1993). The reference for optical information when not specified is from NED (NASA/IPAC Extragalactic Database).

$0108+388$ : the radio galaxy $0108+388$ at $z=0.669$ is extremely interesting because it has extended emission at a distance of about $18^{\prime \prime}(\sim 80 \mathrm{kpc})$ from the compact component, in which a separation velocity of the micro hot-spots of $\sim 0.2 \mathrm{c}$ has been measured by Owsianik et al. (1998). Baum et al. (1990) suggested that the extended radio emission in this object is due to a previous epoch of AGN activity. We confirm that there is extended emission only on the Eastern side of the CSO with a flux density ratio $\sim 25$ between the peak of the detected extended emission and the $3 \sigma$ rms noise (Fig. 1).

If the extended emission is indeed a relic, there should not be any side-to-side asymmetry due to relativistic beaming. Therefore the one-sidedness of the emission in $0108+388$ is

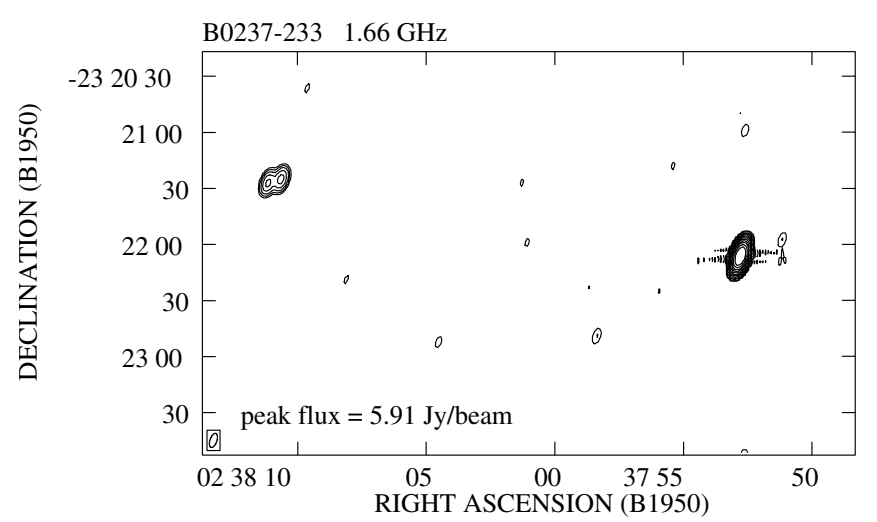

Fig. 2. $0237-233$ at $1.66 \mathrm{GHz}$.

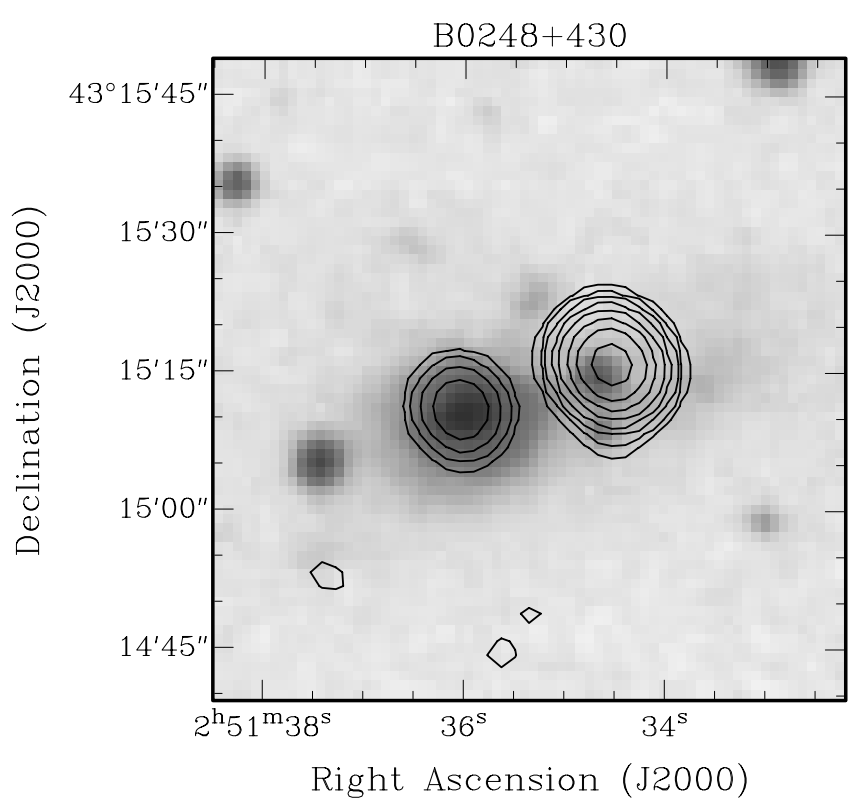

Fig. 3. Radio contours of $0248+430$ at $1.36 \mathrm{GHz}$ superimposed to the gray scale POSS2 image.

puzzling. A possible explanation is that $0108+388$ was not a classical double but was a head-tail radio source, of the kind often seen in rich clusters of galaxies (e.g., O'Dea \& Owen 1985). In this case the one-sided emission would be the relic of a radio tail. However, there is no evidence that $0108+388$ belongs to a rich cluster of galaxies (Stanghellini et al. 1993).

Another possible explanation is that one of the two lobes has already vanished below the threshold of detection. This may happen if the missing lobe expanded faster in the intergalactic medium, and experienced stronger adiabatic losses. It is also possible that the missing lobe faded away faster because of more efficient radiative losses due to a higher magnetic field on that side of the radio source. In principle, a difference in the spectral break due to radiative losses would be expected also if there is a difference in the travel time between the two lobes, in case the source axis is not perpendicular to the line of sight. But the double mas morphology and the lack of relativistic effects suggests the radio axis is mostly perpendicular to the line of sight and the difference in travel time is unlikely to produce a ratio $>25$ between the brightness of the two lobes. 


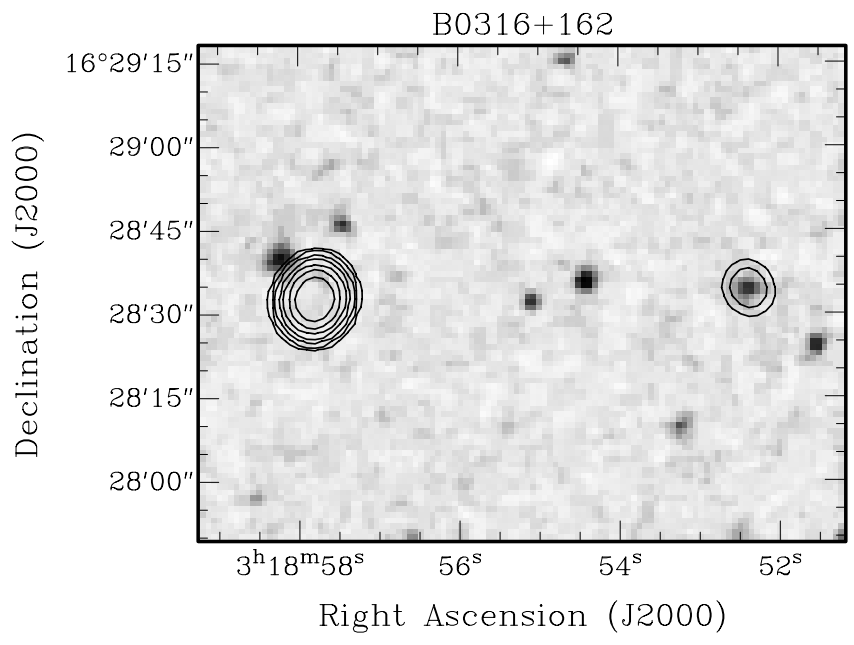

Fig. 4. Radio contours of $0316+162$ at $1.36 \mathrm{GHz}$ superimposed to the gray scale POSS2 image.

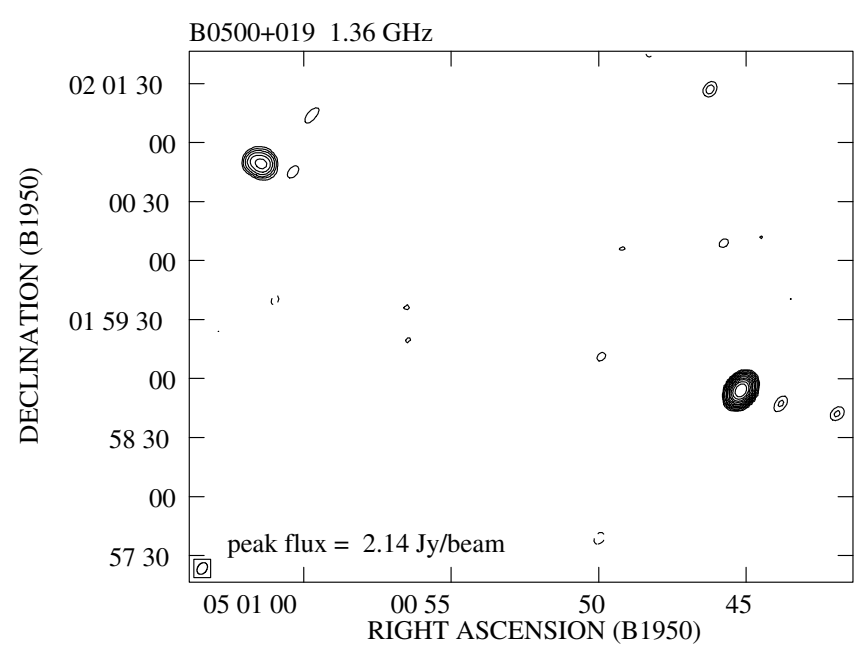

Fig. 5. $0500+019$ at $1.36 \mathrm{GHz}$.

0237-233: a secondary component (Fig. 2) is seen $5^{\prime}$ $(\sim 1.8 \mathrm{Mpc})$ East of the GPS quasar at $z=2.223$. Nothing is visible in the optical POSS2 image at the location of the secondary component. However, the morphology of the secondary component suggests that this is an unrelated double radio source. The horizontal stripes visible close to the dominant emission are artifacts produced in the cleaning and restore procedure.

0248+430: this quasar at $z=1.310$ exhibits a damped Ly $\alpha$ absorbing system apparently due to a galaxy at $z=$ 0.3939. The absorbing galaxy is clearly seen in the POSS2 plate and is coincident with a secondary radio component located 20" ( 120 kpc) East of the GPS quasar (Fig. 3). Thus, it is likely that the secondary radio component is not related to the GPS quasar, and instead is a foreground object.

0316+162: a secondary radio component is present at $3^{\prime}$ west of the faint GPS galaxy. There is an optical object coincident with the secondary component, suggesting that it is an independent radio source (Fig. 4). The projected distance to $0316+162$ is about $1 \mathrm{Mpc}$.

0500+019: Stickel et al. (1996) classify the optical host of this radio source as a quasar, but we consider the identification

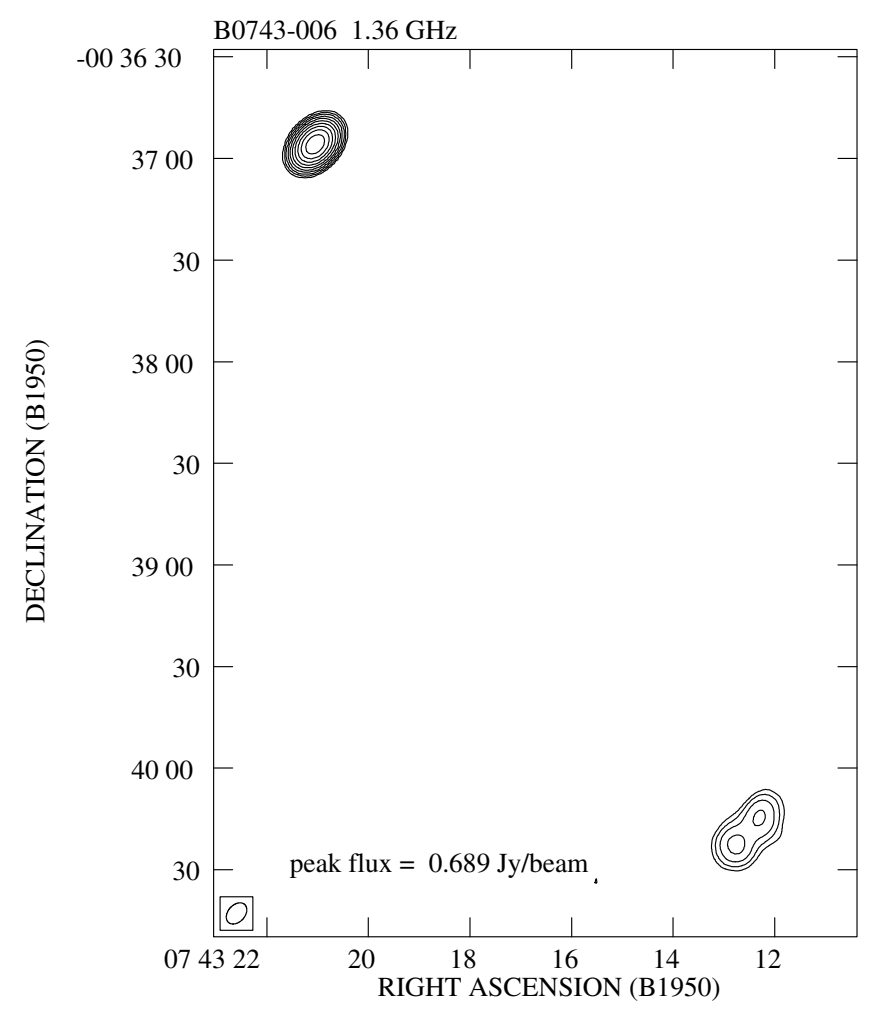

Fig. 6. $0743-006$ at $1.36 \mathrm{GHz}$.

as a galaxy at $z=0.583$ made by De Vries et al. (1995) more reliable. A secondary radio component is present $4^{\prime}(\sim 1.1 \mathrm{Mpc})$ NE of the GPS radio source. No optical counterpart is detected for this secondary component but its large distance from the GPS radio source gives little support to the hypothesis that it is related to it.

$0738+313$ : this object is a quasar at $z=0.631$ with magnitude $m_{V}=16.16$. We find hotspots amidst the lobes on arc second scales which suggests that the AGN is currently active and is resupplying fresh electrons to the extended radio components (Fig. 17). The mas morphology is consistent with a corejet structure with the jet showing a highly boosted knot where it changes direction (helical jet seen in projection?). Detailed radio images of $0738+313$ are presented in Siemiginowska et al. (2003) who discuss the nature of the X-ray emission revealed by Chandra. If we include the extended radio emission, the total projected source size exceeds $300 \mathrm{kpc}$.

0743-006: the optical identification is a quasar at $z=0.994$ of visual magnitude $m_{V}=17.1$. A secondary double component is present $4^{\prime}(\sim 1.4 \mathrm{Mpc}) \mathrm{SW}$ of the GPS source. There is no optical counterpart but its morphology and distance suggest it is an unrelated double radio source.

0941-080: this GPS source is identified with a galaxy with a double nucleus at $z=0.228$. Parsec-scale CSO radio morphology has been found by Stanghellini et al. (1993) and Dallacasa et al. (1998). In our VLA image (Fig. 7), a secondary component is present $20^{\prime \prime}(\sim 50 \mathrm{kpc})$ West of the GPS galaxy. No optical counterpart is detected for this secondary component. It has a spectral index of about -1.2 between 1.36 and 1.67 GHz. X-ray emission is detected both at the position of the main and the secondary components (Siemiginowska, private 


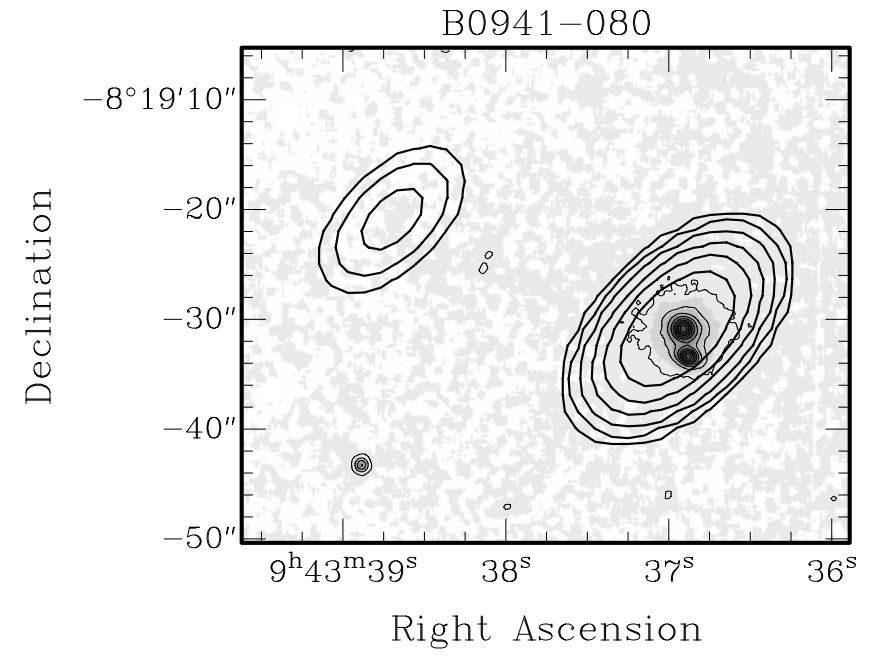

Fig. 7. Radio contours of $0941-080$ at $1.36 \mathrm{GHz}$ superimposed to the NOT optical image (gray scale and thinner contours). First radio contour is $0.5 \mathrm{mJy}$. Peak in the radio image is $2.726 \mathrm{Jy}$.

communication). Given the proximity to the GPS radio source, the steep spectral index, and the lack of an optical counterpart we consider 0941-080 a candidate for a GPS radio galaxy with extended relic emission on the arc second scale.

1127-145: Wehrle et al. (1992) have suggested that this quasar $(z=1.187)$ is a possible compact double because the mas morphology is dominated by 2 components separated by about 4 mas. We believe the mas morphology of this object is better classified as a core-jet with a prominent knot where the jet sharply bends by 45 degrees and continues with additional bends and wiggles even well beyond the arc second scale. The jet can be detected at all the intermediate angular scales from mas to arcsec (Fig. 9) with total extent $25^{\prime \prime}(\sim 150 \mathrm{kpc})$.

We do not see hot-spots on the arc second scale as we do in $0738+313$, but the source is at a higher redshift $(z=1.187)$ and we possibly detect only part of the (moderately beamed) jet. Siemiginowska et al. (2002) report the detection of X-ray emission associated with this jet. The weak radio emission located $3.5^{\prime}(\sim 1.2 \mathrm{Mpc})$ North of the core is likely an unrelated source as the POSS2 image shows that it has an optical counterpart (Fig. 8).

1245-197: this radio source is associated with a faint quasar $\left(m_{V}=\sim 21\right)$ at $z=1.275$. On the $1.36 \mathrm{GHz}$ image (Fig. 10) we see a rather compact radio component $4^{\prime}(\sim 1.4 \mathrm{Mpc})$ West of the dominant GPS radio source. The POSS2 red plate superimposed on the radio contours shows a weak optical feature coincident with this secondary radio emission, therefore it is likely to be an unrelated radio source.

$1345+125$ : this radio source is associated with a galaxy at $z=0.122$ in which a double optical nucleus is clearly detected (Stanghellini et al. 1993; Gilmore \& Shaw 1986). Remarkably, the arcsec scale morphology mimics that seen on the parsec scale, with a southern region bending to the South-West and a weaker, smaller northern component (Fig. 11). The GPS source was first detected in X-rays by ASCA (O'Dea et al. 2000). Newer observations by Chandra reveal X-ray emission coincident with the optical galaxy and extending to the South partly

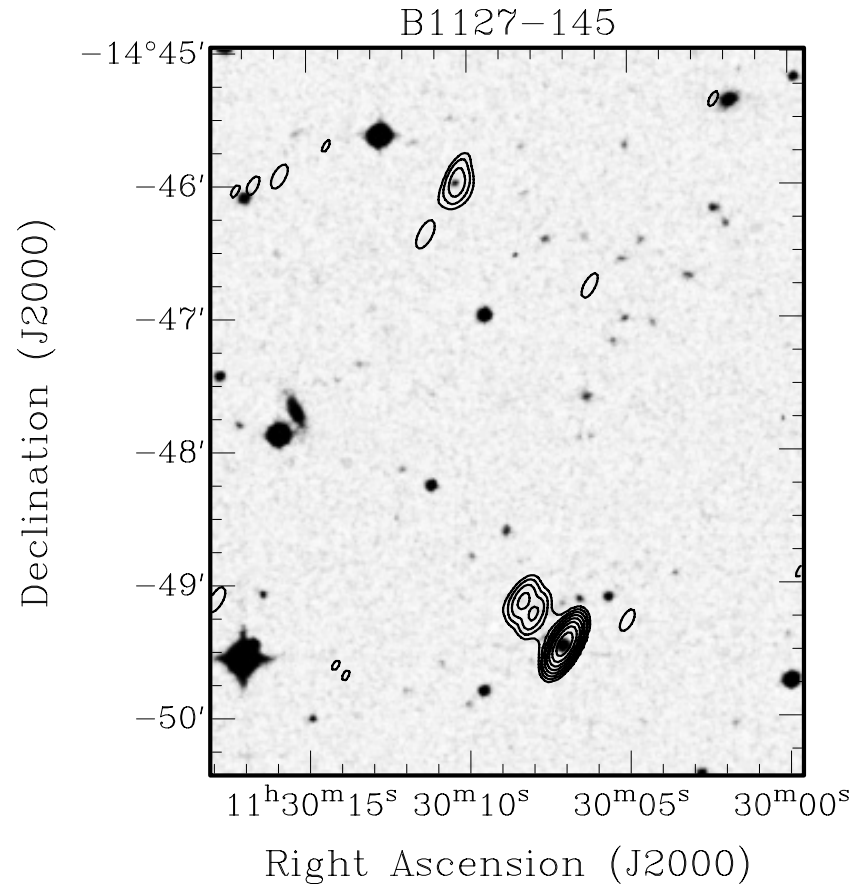

Fig. 8. Radio contours of $1127-145$ at $1.36 \mathrm{GHz}$ superimposed to the gray scale POSS2 image.

coincident with the southern radio emission seen by the VLA (Fig. 3 in Siemiginowska et al. 2004).

Reliable information on the spectral index of the lobes/tails between 1.36 and 1.67 is not possible because the rather poor quality of the $1.67 \mathrm{GHz}$ data does not allow us to properly image the faint low surface brightness extended emission. This is a good example of a GPS radio galaxy with extended emission on scales exceeding 1 arcmin $(\sim 100 \mathrm{kpc})$. However, this radio source has rather unusual properties for a CSO. In addition to the high flux density ratio between the two parsec scale jets/lobes, it has highly polarized components and superluminal motion (Lister et al. 2003). The continuity of the morphology from the small to the large scales is consistent with the hypothesis that there is a continuous supply of energy from the VLBI core to the extended lobes.

$1404+286(O Q 208)$ : this is a compact radio source associated with the bright galaxy Mkn668 with $m_{r}=14.6$ at $z=0.077$ (Stanghellini et al. 1993). This object is one of the closest examples of bright GPS radio sources. It has a somewhat asymmetric CSO morphology on the pc scale (Stanghellini et al. 2001) and there is some indication that it is expanding (Stanghellini et al. 2003). OQ208 is the first radioloud X-ray Compton-thick AGN ever observed. From ASCA and XMM-Newton observations Guainazzi et al. (2004) estimate a colunm density covering the nuclear emission $>9 \times$ $10^{23} \mathrm{~cm}^{-2}$. The obscuring gas is most likely located within the radio hotspot. A further X-ray absorbing system with $N_{\mathrm{H}} \sim$ $10^{21} \mathrm{~cm}^{-2}$ may be associated with gas responsible for free-free absorption of the micro-hotspots.

The detection of a weak (6 mJy) and extended (20", $\sim 20 \mathrm{kpc}$ ) radio halo has been reported by de Bruyn (1990) from WSRT data at $5 \mathrm{GHz}$ around the compact object. However, 


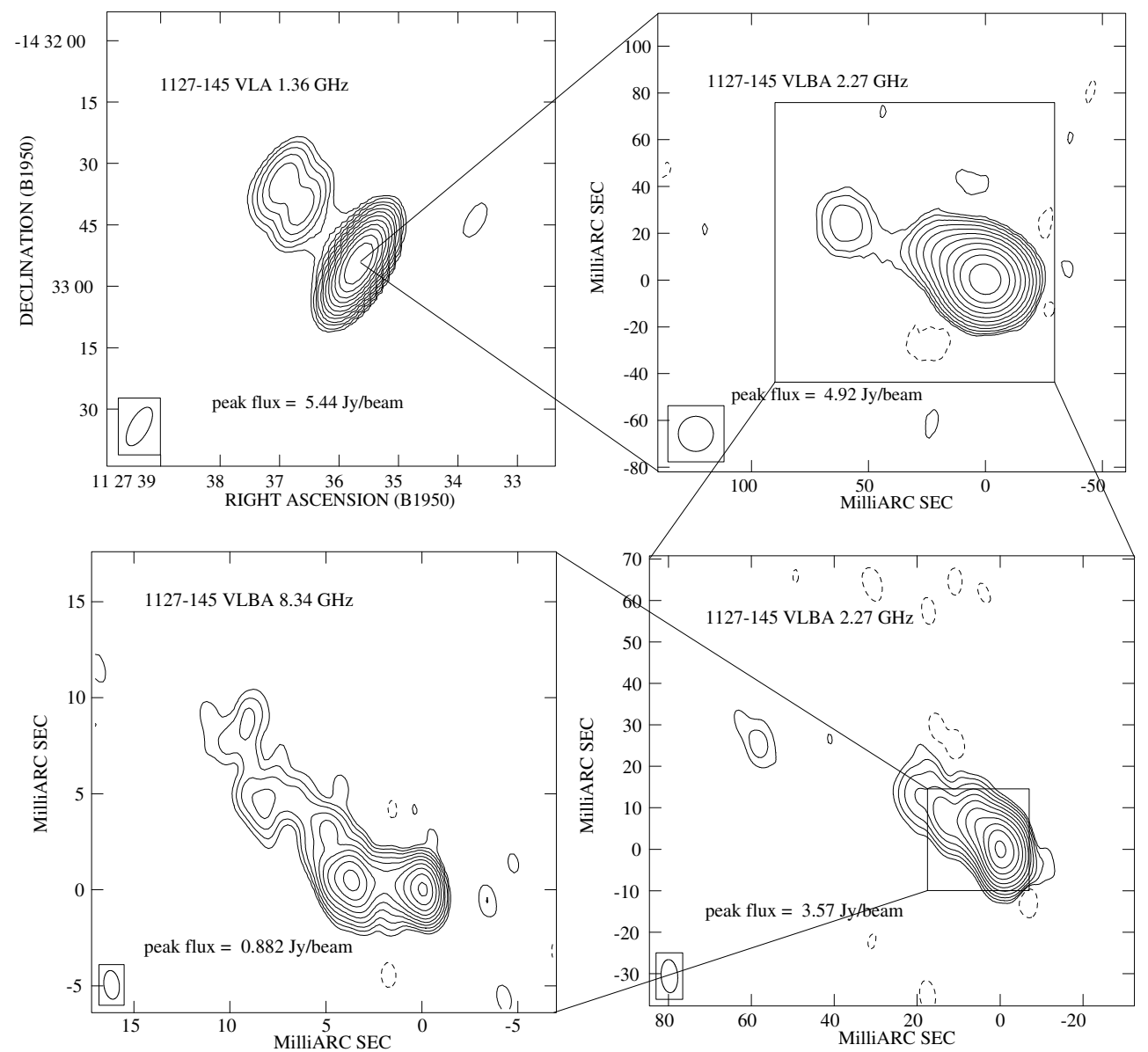

Fig. 9. 1127-145 from mas to arcsecond scale.

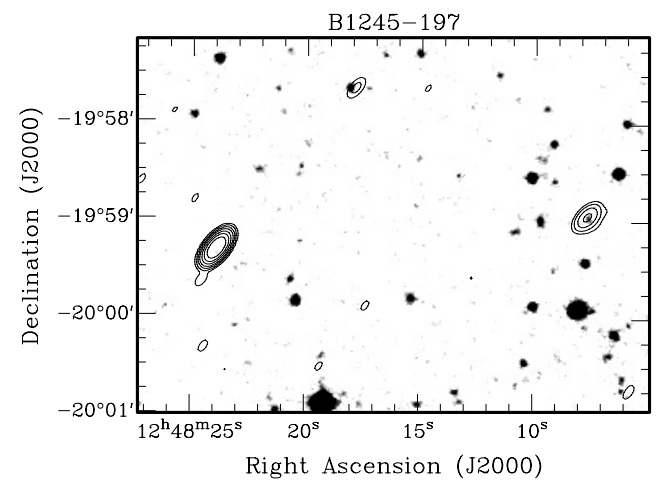

Fig. 10. Radio contours of $1245-197$ at $1.36 \mathrm{GHz}$ superimposed to the gray scale POSS2 image.

we do not see any evidence for the extended emission in our VLA images at 1.36 or $1.67 \mathrm{GHz}$ (Fig. 12). Radio emission seen in lower resolution observations may be resolved out with higher resolution, though our VLA observations should be sensitive to angular scales up to a couple of arcminutes.

A secondary component with no optical counterpart is detected $3^{\prime}(\sim 180 \mathrm{kpc}) \mathrm{NE}$ of the GPS radio galaxy. Its nature is uncertain, but we suspect it is probably an unrelated object.

$1518+047:$ it is one of the rare quasars $(z=1.296)$ which have a CSO morphology on the pc scale (Dallacasa et al. 1998). The NVSS image suggests the presence of extended emission around this GPS radio source but the VLA B array image shows an isolated secondary component about $1^{\prime}(\sim 360 \mathrm{kpc}) \mathrm{SW}$ of the GPS radio source (Fig. 13). There is no optical counterpart on the POSS2 plate and it is unclear if this component is associated with the GPS source.

2008-068: this CSO is identified with a galaxy with unknown redshift. Using its optical $R$ magnitude of 21.3 and the GPS galaxy Hubble diagram (O'Dea et al. 1996) we can estimate $z \sim 0.7$. This case is very similar to the previous one. The NVSS indicates extended emission; however, the VLA B array image reveals an isolated secondary compact component at about $50^{\prime \prime}(\sim 250 \mathrm{kpc})$ to the SE of the main one, with no optical counterpart (Fig. 14). The spectral index between 1.36 and 1.67 of this secondary component is slightly inverted so it is probably an unrelated radio source.

2128+048: this CSO is associated with a very red galaxy (magnitude $m_{r}=23.3, m_{i}=21.85$ ) by Biretta et al. (1985) with redshift 0.990 (Stickel et al. 1994). We detect a slightly resolved radio component $4^{\prime}(\sim 1.4 \mathrm{Mpc}) \mathrm{SE}$ of the GPS radio galaxy with no optical counterpart (Fig. 15).

2134+004: the quasar 2134+004 has a double morphology at mas scale which has been interpreted in the context of a corejet structure (Stanghellini et al. 2001). On the arc second scale it shows extended emission South of the dominant compact component, connected to and likely associated with the GPS source (Fig. 16), and an isolated knot at 1.5' ( 540 kpc) from the core, 


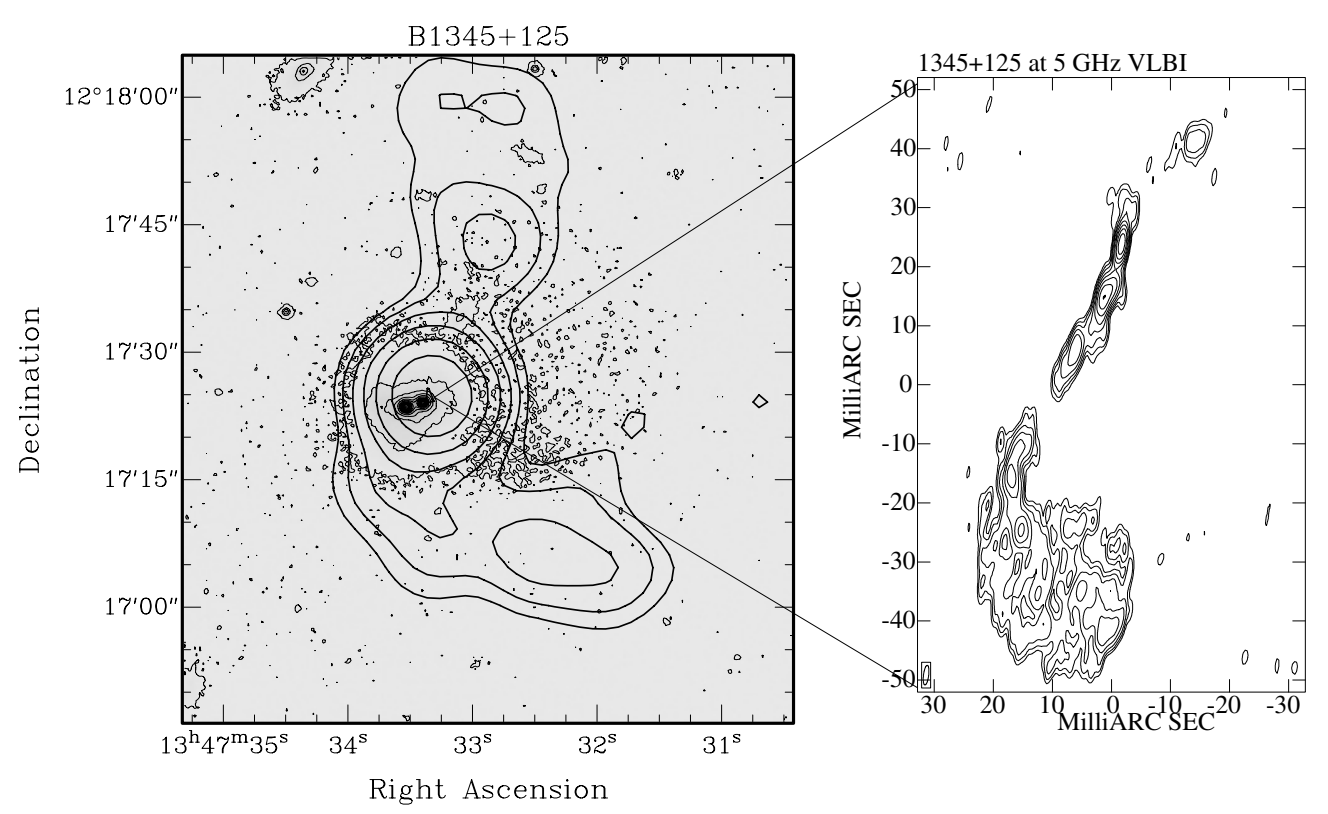

Fig. 11. Radio contours of $1345+125$ superimposed to the NOT optical image (gray scale image and thinner contours). First radio contour is $1 \mathrm{mJy}$. The peak in the image is $64.8 \mathrm{mJy}$. A point like component of $5.2 \mathrm{Jy}$ has been subtracted at the peak position to enhance the visibility of the diffuse emission around the core.

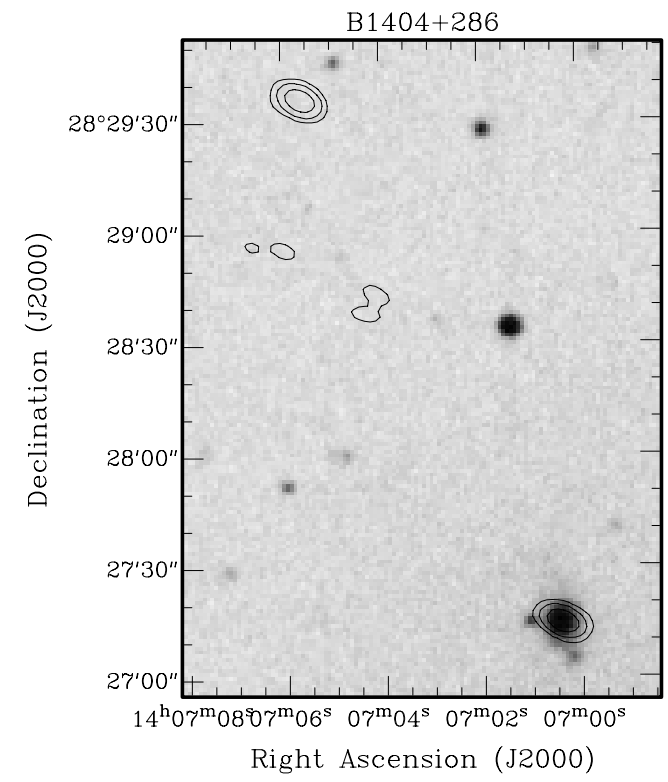

Fig. 12. Radio contours of $1404+286$ at $1.36 \mathrm{GHz}$ superimposed to the gray scale POSS2 image

of uncertain interpretation, and without an optical counterpart on the POSS2 image.

\section{Discussion}

We observed all 33 GPS radio sources in the 1 Jy complete sample defined by Stanghellini et al. (1998). We find 16 objects showing some extended radio emission closer than either tens of arcsec or a few arcmin. In general, such additional components are much weaker than the GPS source.

Nine sources exhibit radio emission closer than 2 arcmin from the bright compact GPS source, corresponding to a distance of about half a Mpc for redshifts above 0.2. In six of them, 0108+388, 0738+313, 0941-080, 1127-145 and $1345+125$, and $2134+004$ the extended emission seems related to the GPS source based on the morphological and spectral index information, possibly due to an earlier episode of activity. For the remaining three sources, with additional emission at a projected distance ranging from about $120 \mathrm{kpc}$ to about $360 \mathrm{kpc}$, a physical connection can be excluded in the case of $0248+430$ due to the presence of another optical identification for the confusing source, and of 2008-068 due to the flat spectral index of the secondary component. It is unclear how to interpret the additional components in the field near 1518+047. It seems likely that it is an unrelated field source, given that it is unresolved by our observations.

The weakest secondary component in these 9 fields of 2 arcmin radius, is the $9.3 \mathrm{mJy}$ component East of $0108+388$.

Based on source counts at $1.4 \mathrm{GHz}$, there is a $\sim 5 \%$ probability of finding a radio source brighter than $10 \mathrm{mJy}$ within 2 arcmin from our GPS sources (Prandoni, private communication). Thus, we might expect to find about 2 such coincidences by chance.

The radio source $1404+286$ has a secondary component at about 3 arcmin distance, but since it is at a low redshift, the corresponding linear distance is $180 \mathrm{kpc}$ only. A connection with the main component cannot be completely excluded, but it is unresolved and in this case we prefer the intrepretation as an unrelated field source.

Six sources (0237-233, 0316+162, 0500+019, 0743-006, 1245-197 and 2128+048) have possible secondary components at projected distances exceeding $1 \mathrm{Mpc}$ making the physical connection very unlikely, since it would imply a total source size two times larger if we consider an invisible (faded away?) counterpart on the other side of the newly active GPS nucleus. 

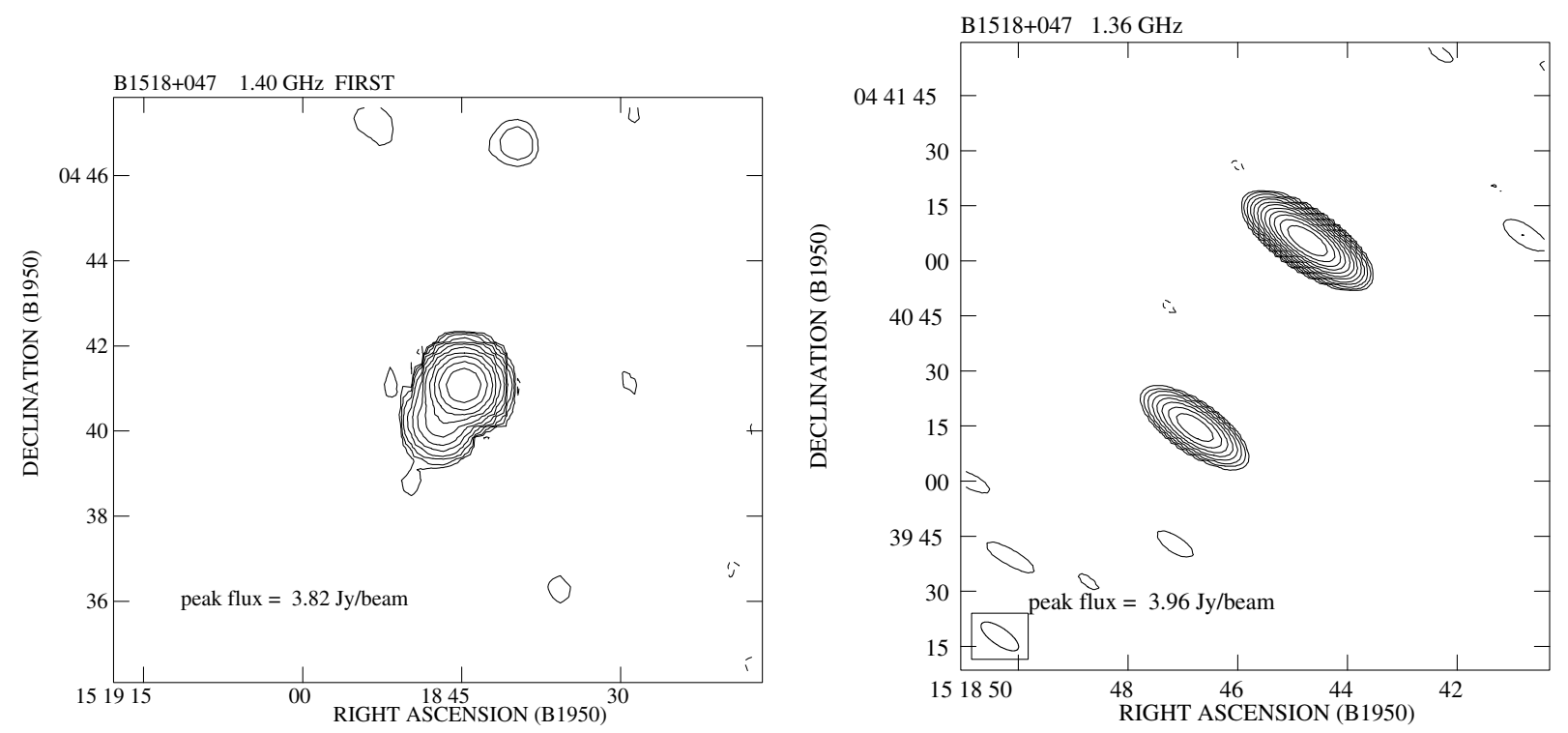

Fig. 13. $1518+047$ from the FIRST (left) and from present data (right).
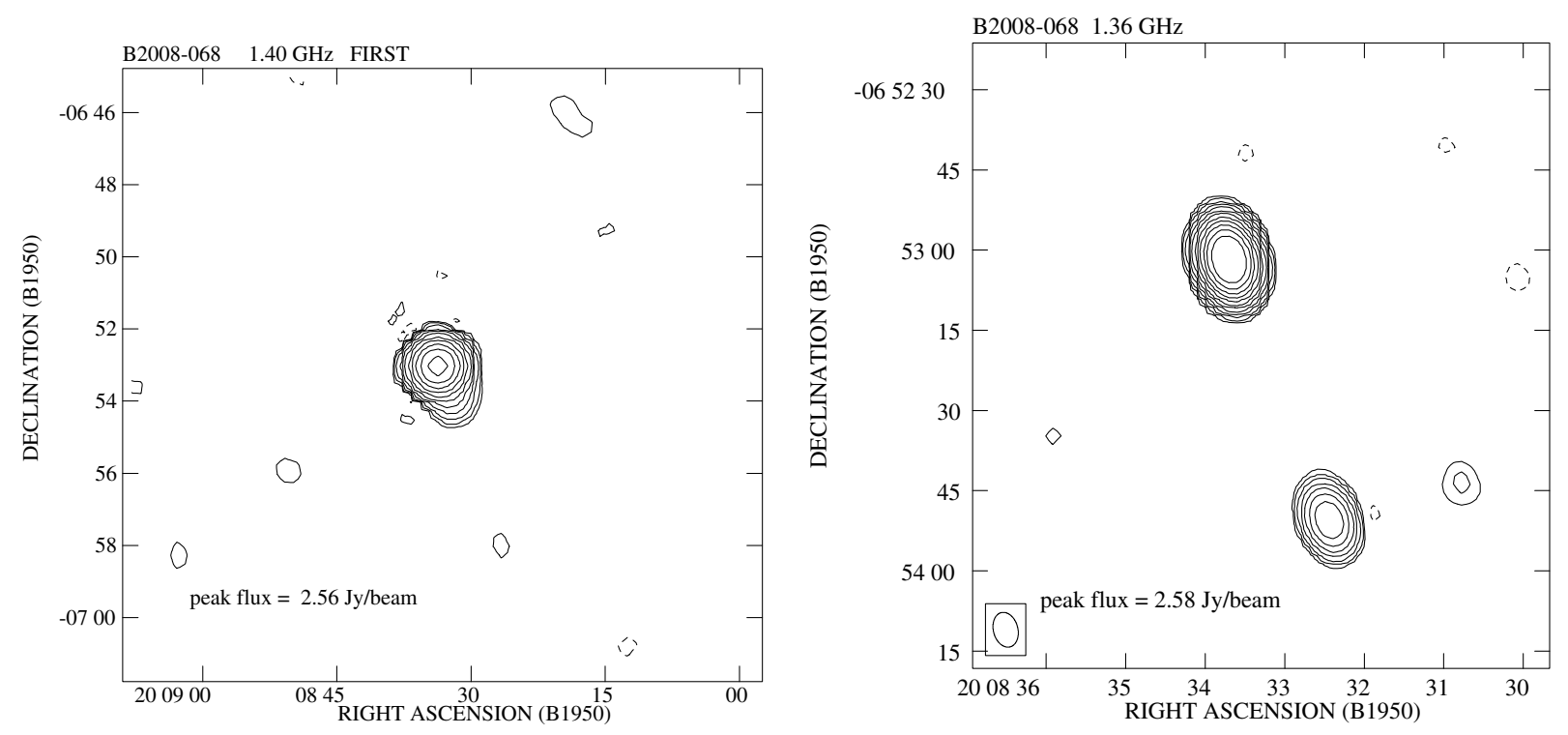

Fig. 14. 2008-068 from the FIRST (left) and from present data (right).

In Fig. 17 we show how $0738+313$ (at $z=0.631$ ) would appear at redshifts of $z=2$ and $z=3$, keeping constant sensitivity of the observations (the change of the angular size has not been considered since at high redshift the angular size changes very smoothly). At $z=2,0738+313$ would appear as a strong radio source with a secondary weak component $30^{\prime \prime}$ in the north direction, and another barely visible on the other side of the strong component. At $z=3$ there would be no clear evidence of additional radio emission, just a hint of the northern hotspot emission which could be easily interpreted as a peak in the noise. Due to the redshift distribution of the 14 GPS quasars of our sample, the extended emission revealed around $0738+313$ (which is the closest quasar of our sample) would be impossible to detect in the vast majority of them (Fig. 18). This indicates that the extended, low surface-brightness lobes of a classical double may be below the detection limits of our observations, and only the brightest components (the GPS core and one or two isolated components, possibly the hot-spots or knots in a jet) are visible in our images. Deeper observations are needed to search for fainter emission connecting the GPS source and the secondary components.

In Fig. 18 we plot the flux density we have detected (and upper limits) from the extended emission (assuming the extended sources cover an area of ten times the beam areas) as a function of redshift. In the diagram, the regions usually populated by FRI and FRII-type radio sources (e.g., Ledlow \& Owen 1996) are also marked. This illustrative example shows that we cannot rule out the possibility that the GPS sources contain extended FRI and even FRII emission that remains undetected because our sensitivity rapidly decreases with redshift. 


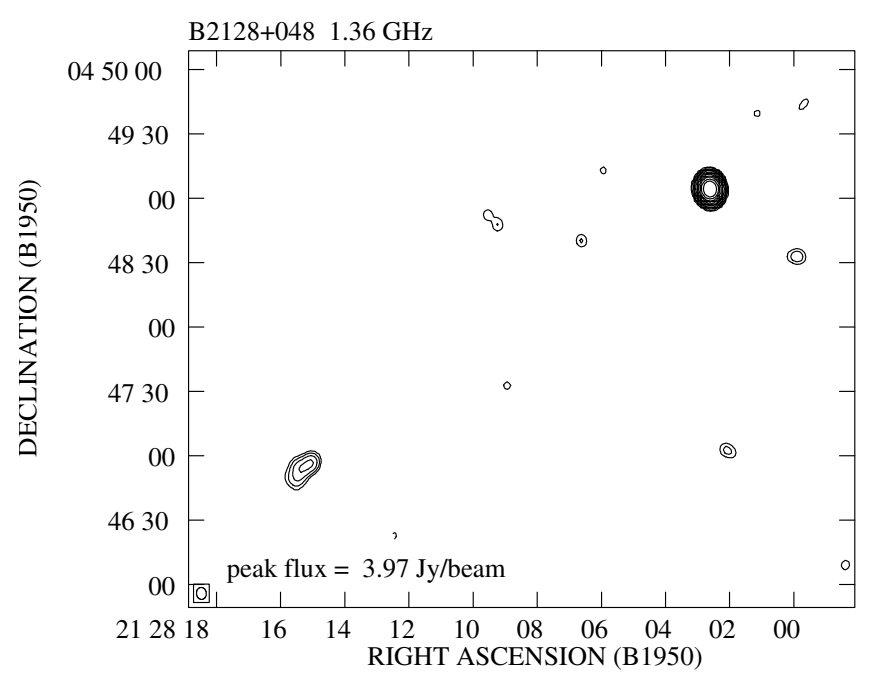

Fig. 15. Radio image of $2128+048$ at $1.36 \mathrm{GHz}$.

In this somewhat heterogeneous picture, three galaxies (out the 19 galaxies in our sample) appear to have extended emission:

- $1345+125$ where there is continuity between the small and the large scale with a (twin) jet progressively diffusing into a low surface brightness tail;

- 0108+388 in which the youth of the small scale radio emission has been confirmed by the measure of the advance speed of the micro-hotspots (Owsianik et al. 1998), and there is resolved, steep spectrum old emission on the large scale. This is a likely case of recurrent activity as already proposed by Baum et al. (1990);

- 0941-080 where X-rays have been detected from the additional component, complicating the interpretation.

On the other hand, the three quasars have a core-jet morphology on the small scale while on the large scale we see either an FR-II type radio source $(0738+313)$ or the continuation of the small scale jet (1127-145) or a more complex structure (2134+004).

Why do we see different relationships between the extended emission and the compact GPS source? It has been clear for a long time that the selection of sources on the basis of a peaked spectrum produces a somewhat heterogeneous sample of objects (O'Dea et al. 1991). So we should not be surprised to find different relationships among the sources discussed here.

\subsection{Extended emission in GPS galaxies}

Among the 19 galaxies of the complete sample, the only clear case where the recurrent hypothesis can be applied is that of $0108+388$, whose youth has been confirmed by the kinematic age measurement (Owsianik et al. 1998). A similar case could be 0941-080, but the kinematic age is not yet available and we know little about the properties of the additional component.

Finally, the source $1345+125$ shows evidence for continuity between the small and large scale emission, which, if confirmed, would suggest that the extended emission is not the relic of past activity.
The total fraction of GPS galaxies with extended emission is therefore between $5-16 \%$ (1-3 out of 19). We can therefore conclude that in general, the presence of large scale emission associated with GPS galaxies is quite rare, and all but one of the cases found so far still are subject to the possibility that they could arise from chance projection effects rather than a physical connection. In the context of the recurrent activity model, all this suggests that the time scale between subsequent bursts is in general longer than the radiative lifetime of the radio emission from the earlier activity $\left(\sim 10^{8} \mathrm{yr}\right)$.

In several sources we may be witnessing ongoing (or at least recent) transport of radio plasma from the core to the extended structure. In the case of the GPS quasar $0738+313$, with a core-jet mas morphology, we see the hotspots on arcsecond scales which suggest that there is a continuous supply of fresh electrons from the nucleus. In the quasar 1127-145 which also has core-jet mas morphology, we see radio emission from the mas to the arcsecond scale. Again, this suggests the existence of a continuous flow of relativistic electrons from the core to the arcsecond scale jet. The quasar 2134-004 exhibits mas morphology consistent with a core-jet source suggesting transport of radio plasma to larger scales. However, in this source there is no evidence on intermediate scales for a continuous connection.

\subsection{The nature of GPS quasars}

The relationship between GPS galaxies and quasars has been a longstanding question. Are the GPS quasars the beamed counterparts of the GPS radio galaxies as predicted in Unified Scheme Models (e.g. Urry \& Padovani 1995)? Or are they unrelated objects simply having similar radio spectra? The idea that the GPS quasars and radio galaxies are different phenomena has been discussed by Snellen (1997) and Stanghellini et al. (2001). Based on the radio morphological evidence presented here on both the small and large scales, we suggest that the extended emission we have detected in the GPS quasars is currently being supplied with energy by the nucleus. A plausible hypothesis is that the GPS quasars exhibiting a core-jet or complex morphology on the mas scale are indeed "extended" (namely with lobes larger than tens/hundreds of kpc) but coredominated radio sources whose redshifts are sufficiently high that most (if not all) of the the large scale structure is below our detection threshold.

This would imply that GPS quasars with core-jet mas morphology represent an intermediate population of objects morphologically more similar to the common flat spectrum radio sources which dominate the radio surveys at $\mathrm{cm}$ wavelengths than to the CSO/galaxy phenomenon. If we assume that their jets are oriented with an angle to the line of sight intermediate between flat spectrum quasars and radio galaxies, GPS quasars would play the same role as the steep spectrum radio quasars in the extended sources.

More generally, what causes the GPS spectral shape in these quasars?

There is certainly evidence for moderate beaming in the GPS quasars (e.g., O'Dea 1998). There is evidence that there is 


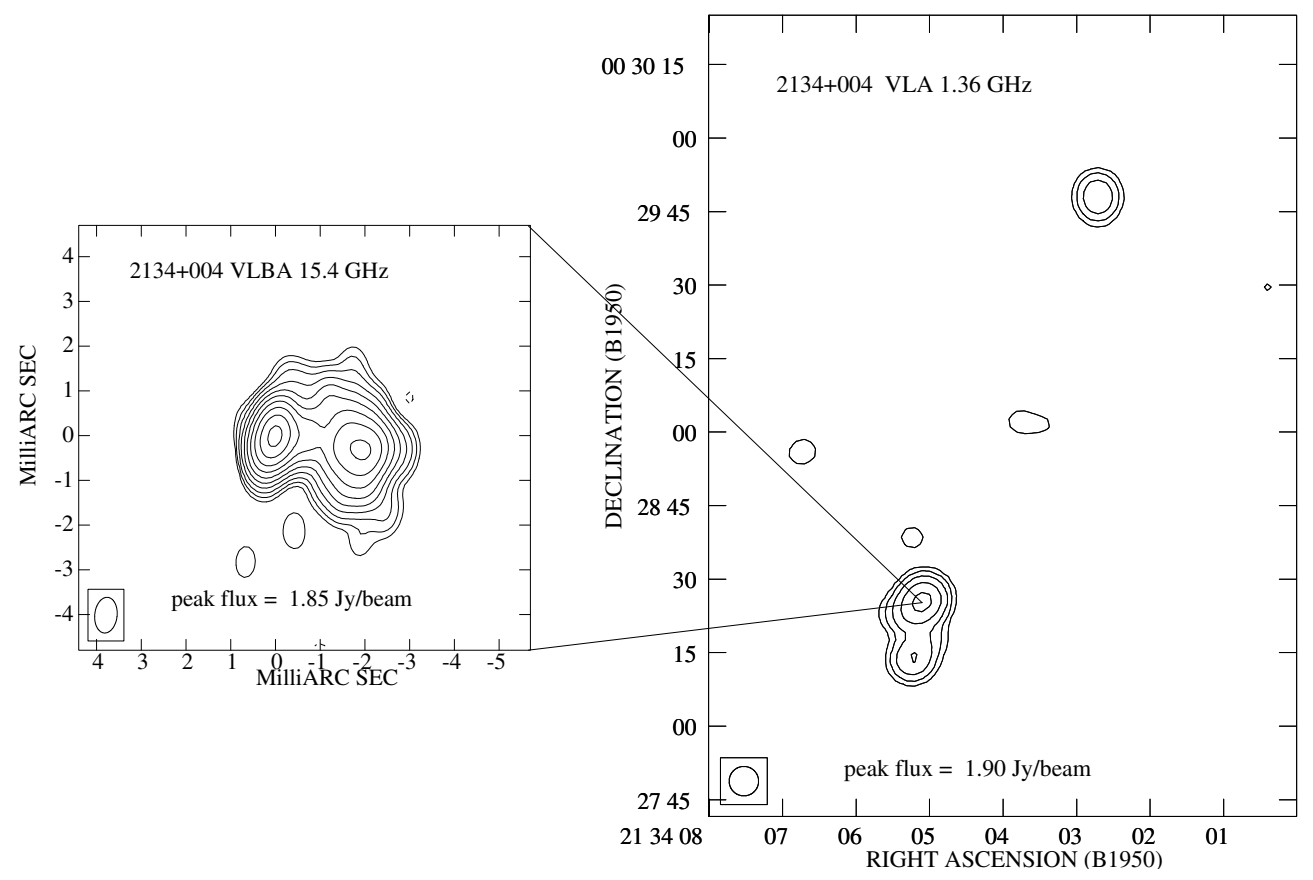

Fig. 16. 2134+004 at mas (left) and arcsecond resolution (right). In the VLA image most of the core flux density has been subtracted to enhance the visibility of the diffuse emission.
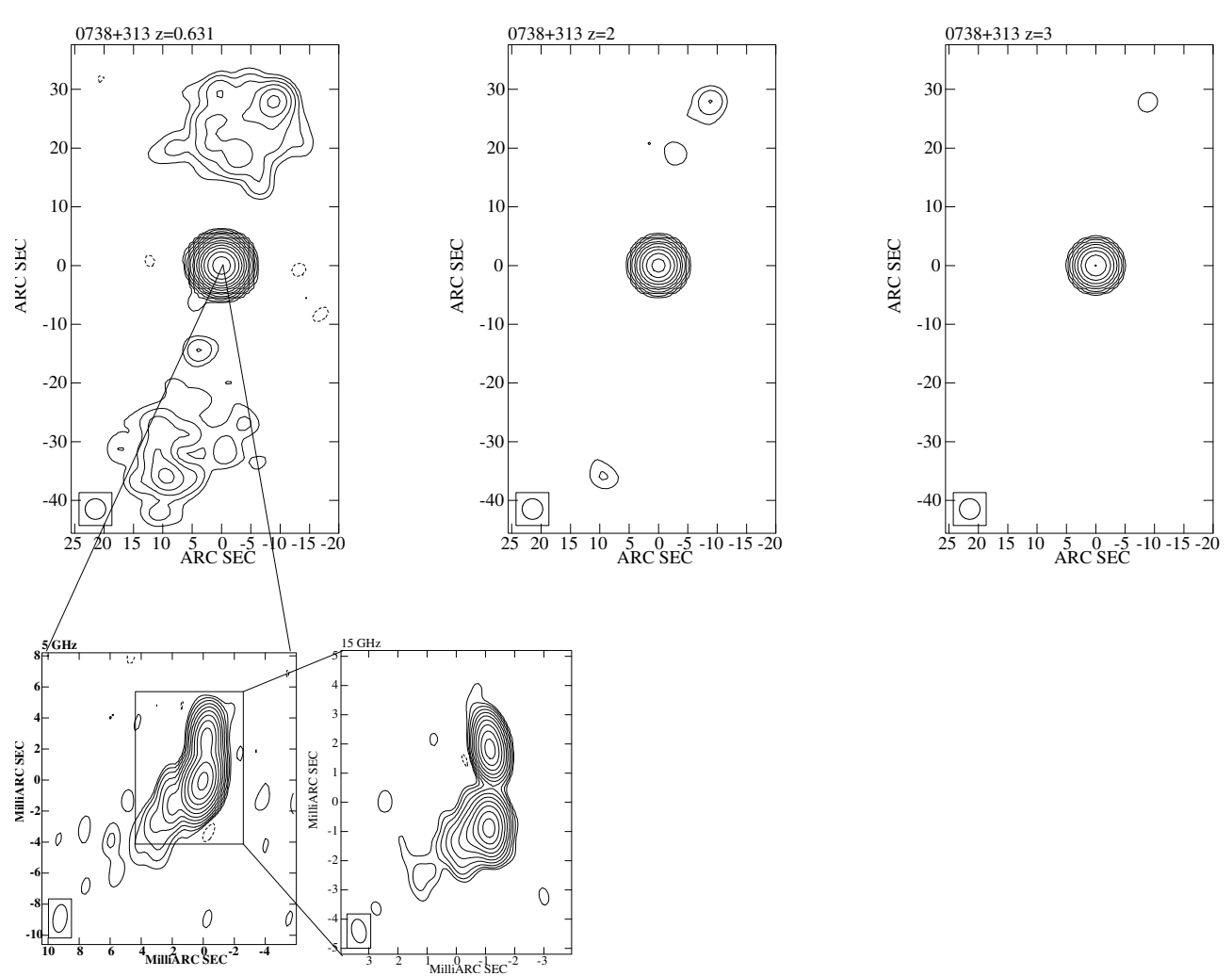

Fig. 17. The effect of redshift on extended emission detectability. Left image is $0738+313$ at $z=0.631$, center image is how it would appear at $z=2$, right image is how it would appear at $z=3$. The dependence of angular size with redshift has not been taken into consideration because at these high redshifts angular size does not depend strongly on redshift.

larger variability in the GPS quasars than in GPS radio galaxies (e.g., Stanghellini 1999; Fassnacht \& Taylor 2001; Aller et al. 2002). In particular, $0738+313,1127-145$, and 2134+004 (in which we find extended emission) have been shown by Aller et al. (2002) to be variable. Nevertheless, they usually maintain their convex spectrum during the variability, although the present shape of the radio spectrum for $1127-145$ is flat and would not be classified as a GPS radio source today.

Furthermore, the GPS quasars have higher polarization than the radio galaxies (generally unpolarized) but are 


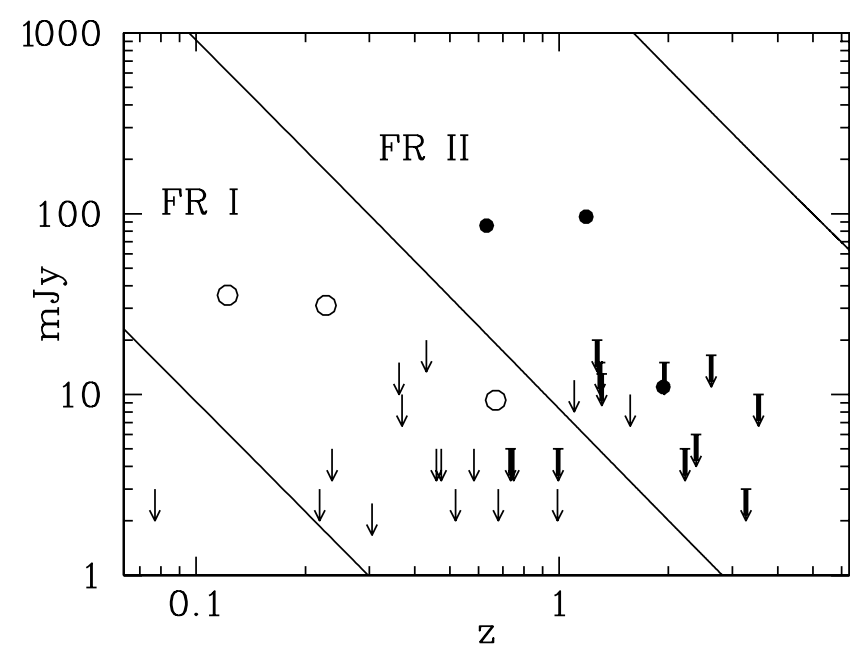

Fig. 18. Flux density at $1.36 \mathrm{GHz}$ of extended emission versus redshift. Continuous lines approximately separate regions of FRI and FRII radio sources, filled circles are quasars, empty circle are galaxies, thick arrows are higher limits for quasars, thin arrows are higher limits for galaxies (see Sect. 4).

less polarized than flat spectrum quasars (e.g., Stanghellini 1999; Aller et al. 2002). Hence, it seems plausible that the GPS quasars are not quite as strongly beamed as the "flat spectrum" quasars, a possible further indication of an intermediate orientation.

GPS radio sources are almost evenly optically identified with galaxies and quasars in the complete sample selected by Stanghellini et al. (1998). Does this apply to other samples? Dallacasa et al. (2000) selected a sample of extreme GPS radio sources with convex radio spectra peaking at frequencies above a few $\mathrm{GHz}$, and with flux density limit at $5 \mathrm{GHz}$ of $300 \mathrm{mJy}$. They call these radio sources High Frequency Peakers (HFPs, see also Dallacasa 2003). That sample extends to higher turnover frequencies the samples of Compact Steep Spectrum (CSS) and GHz Peaked Spectrum (GPS) radio sources. Dallacasa et al. (2002a) discuss the optical identification of the objects in the sample and they find that $26 \%$ are galaxies and $74 \%$ are quasars or stellar objects.

Fanti et al. (2001) selected a new sample of low/intermediate luminosity CSS radio sources from the B3 sample. The fraction of quasars is very low and the vast majority of the identifications are with galaxies or empty fields. Dallacasa et al. $(2002 b, c)$ observed 46 of these low luminosity CSS sources with MERLIN, EVN, and the VLBA. They find that 36 are CSO or MSO, 2 are core-jet, and 8 of uncertain or irregular morphology. Thus, the core-jet morphology on the small scale typically found in flat spectrum quasars/blazars is found in about half of bright GPS quasars and only in some faint CSSs. Moreover there is a tendency that the incidence of quasars and/or core-jet morphologies decreases with decreasing turnover frequency (i.e., increasing size), and possibly decreasing brightness.

This is consistent with the view that GPS spectrum in quasars and galaxies originate from intrinsically different emitting regions: micro lobes/hotspots with a large range in sizes in galaxies, while in the quasars the emitting region tends to be more compact and closer to the core. The structure in the GPS quasars may also be simpler with most of the emission coming from one or a few knots in a moderately beamed jet. These knots may correspond to the location of a shock front where the electrons are re-accelerated or a turn in a helical jet where the bulk flow is locally pointing toward the observer, enhancing its brightness by moderate relativistic beaming. This difference with respect to the other flat spectrum radio sources may be also the cause for the longer variability time scale of the flux density reported by Aller et al. (2002)

We remark that some GPS quasars may be young/ frustrated/recurrent radio sources seen with the AGN axis aligned toward us. The mas radio morphology is fundamental to allow us to test this hypothesis. However, this may be difficult to achieve in practice since unbeamed lobe emission may be hard to see in these distant sources against the beamed jet/knot emission unless very high dynamic range images are obtained. Generally, if we do not see a CSO morphology in the quasars, a simple (and likely) explanation is that the quasar is an intrinsically large, old and active radio source seen along the radio axis. The extended emission may not be seen in many cases, since it will be below our detection limit at the redshift of the quasar. On the other hand, CSO morphologies in quasars, or at least mini-lobe dominated structures may be signatures of an intrinsically small and young radio source.

Thus, we suggest that GPS samples are contaminated by radio sources (mostly quasars) that have nothing to do with the youth/frustrated/recurrent scenarios. A combination of radio spectrum, CSO mas radio morphology and the lack of extended structure should be used to select young quasars.

\section{Summary}

We searched for extended arcsecond scale radio emission in a sample of 33 GPS radio sources. We find extended emission which we consider likely to be associated with the GPS source in 6 objects. Three of these have CSO morphology and three have core-jet morphology. At this point $0108+388$ remains the only strong candidate for a "re-born" GPS radio source, with the possible addition of 0941-080.

We suggest that GPS radio sources with a core-jet mas morphology (almost exclusively quasars) are likely to be large radio sources seen in projection and moderately beamed towards us. Extended emission is possibly not detectable in some of these because they are at high redshift where the extended emission is below our detection threshold. GPS quasars are more variable in radio flux density and more polarized than commonly assumed, and may represent an intermediate population between the intrinsically small GPS galaxies and the flat spectrum radio quasars. Quasars with core-jet or complex morphology tend to be more numerous in samples of brighter and smaller radio sources, and dominate the bright HFP sample, while they are rare in the intermediate luminosity B3-VLA CSS sample.

We conclude there is evidence that GPS/CSS quasars with a core-jet and/or complex mas morphology and CSOs are unrelated by unification, but have similar radio spectra. Any inferences regarding e.g., radio source evolution based on 
complete samples of GPS/CSS radio sources should take into consideration this contamination from the quasars and should be limited to CSOs when information on morphology is available or limited to galaxies if mas morphologies are unknown.

Acknowledgements. Part of this work has been done during visits of C.S. at the Space Telescope Science Institute, Baltimore, under the STScI Collaborative Visitor Program. The VLA is operated by the US National Radio Astronomy Observatory which is operated by Associated Universities, Inc., under cooperative agreement with the National Science Foundation. The Westerbork Synthesis Radio Telescope is operated by the Netherlands Foundation for Research in Astronomy (NFRA) which is financially supported by the Netherlands organization for scientific research (NWO) in the Hague. We have made use of the NASA/IPAC Extragalactic Database, operated by the Jet Propulsion Laboratory, California Institute of Technology, under contract with NASA. This research has made use of the United States Naval Observatory (USNO) Radio Reference Frame Image Database (RRFID).

\section{References}

Alexander, P. 2000, MNRAS, 319, 8

Aller, M. F., Aller, H. D, Hughes, P. A., \& Plotkin, R. M. 2002, in Proc. of the 6th European VLBI Network Symposium, ed. E. Ros, R. W. Porcas, A. P. Lobanov, \& J. A. Zensus, MPIfR, Bonn, Germany, 131

Baum, S. A., O’Dea, C. P., Murphy, D. W., \& de Bruyn, A. G. 1990, A\&A, 232, 19

Biretta, J. A., Schneider, D. P., \& Gunn, J. E. 1985, AJ, 90, 2508

van Breugel, W., Miley, G., \& Heckman, T. 1984, AJ, 89, 5

Carvalho, J. C. 1994, A\&A, 292, 392

Carvalho, J. C. 1998, A\&A, 329, 845

Condon, J. J., Cotton, W. D., Greisen, E. W., et al. 1998, AJ, 115, 1693

Dallacasa, D. 2003, PASA, 20, 79

Dallacasa, D., Bondi, M., Alef, W., \& Mantovani, F. 1998, A\&AS, 129,219

Dallacasa, D., Stanghellini, C., Centonza, M., \& Fanti, R. 2000, A\&A, 363,887

Dallacasa, D., Falomo, R., \& Stanghellini, C. 2002a, A\&A, 382, 53

Dallacasa, D., Tinti, S., Fanti, C., et al. 2002b, A\&A, 389, 115

Dallacasa, D., Fanti, C., Giacintucci, S., et al. 2002c, A\&A, 389, 126

de Bruyn, A. G. 1990, in Proc. Dwingeloo Workshop, Compact Steep Spectrum and GHz Peaked Spectrum Radio Sources, ed. C. Fanti, R. Fanti, C. P. O’Dea, \& R. T. Schilizzi (Bologna: Istituto di Radioastronomia), 105

de Vries, W. H., Barthel, P. D., \& Hes, R. 1995, A\&AS, 114, 259

De Young, D. S. 1993, ApJ, 402, 95

Fanti, C., Fanti, R., Dallacasa, D., et al. 1995, A\&A, 302, 317

Fanti, C., Pozzi, F., Dallacasa, D., et al. 2001, A\&A, 369, 380
Fassnacht, C. D., \& Taylor, G. B. 2001, AJ, 122, 1661

Fey, A. L., Clegg, A. W., \& Fomalont, E. B. 1996, ApJS, 105, 299

Gilmore, G., \& Shaw, M. A. 1986, Nature, 321, 750

Guainazzi, M., Siemiginowska, A., Rodriguez-Pascual, P., \& Stanghellini, C. 2004, A\&A, 421, 461

Ledlow, M. J., \& Owen, F. N. 1996, AJ, 112, 9

Lister, M. L., Kellermann, K. I., Vermeulen, R. C., et al. 2003, ApJ, 584,135

O’Dea, C. P., \& Owen, F. N. 1985, AJ, 90, 927

O’Dea, C. P., Stanghellini, C., Baum, S. A., \& Charlot, S. 1996, ApJ, 470,806

O’Dea, C. P. 1998, PASP, 110, 493

O'Dea, C. P., de Vries, W. H., Worrall, D. M., Baum, S. A., \& Koekemoer, A. 2000, AJ, 119, 478

Owsianik, I., Conway, J. E., \& Polatidis, A. G. 1998, A\&A, 336, L37

Perley, R. A. 1982, AJ, 87, 859

Phillips, R. B., \& Mutel, R. L. 1982, A\&A, 106, 21

Readhead, A. C. S., Taylor, G. B., Xu, W., et al. 1996a, ApJ, 460, 612

Readhead, A. C. S., Taylor, G. B., Pearson, T. J., \& Wilkinson, P. N. 1996b, ApJ, 460, 634

Siemiginowska, A., Bechtold, J., Aldcroft, T. L., et al. 2002, ApJ, 570, 543

Siemiginowska, A., Stanghellini, C., Brunetti, G., et al. 2003, ApJ, 595,643

Siemiginowska, A., Aldcroft, T. L., Bechtold, J., Elvis, M., \& Stanghellini, C. 2004, in X-ray and radio connection, Proc. of the meeting held in Santa Fe in Feb. 2004

Snellen, I. A. G. 1997, Ph.D. Thesis, Univ. of Leiden

Snellen, I. A. G., Schilizzi, R. T., Miley, G. K., et al. 2000, MNRAS, 319,445

Snellen, I. A. G., Mack, K.-H., Schilizzi, R. T., \& Tschager, W. 2003, PASA, 20, 38

Stanghellini, C. 1999, MmSAI, 70, 117

Stanghellini, C. 2003, PASA, 20, 118

Stanghellini, C., Baum, S. A., O'Dea, C. P., \& Morris, G. B. 1990, A\&A, 233, 379

Stanghellini, C., O’Dea, C. P., Baum, S. A., \& Laurikainen, E. 1993, ApJS, 88, 1

Stanghellini, C., O’Dea, C. P., Baum, S. A., et al. 1997, A\&A, 325, 943

Stanghellini, C., O'Dea, C. P., Dallacasa, D., et al. 1998, A\&AS, 131, 303

Stanghellini, C., Dallacasa, D., O’Dea, C. P., et al. 2001, A\&A, 377, 377

Stanghellini, C., Dallacasa, D., O’Dea, C. P., et al. 2002, NewAR, 46, 291

Stickel, M., Meisenheimer, K., \& Kühr, H. 1994, A\&AS, 105, 211

Stickel, M., Rieke, M. J., Rieke, G. H., et al. 1996, A\&A, 306, 49

Urry, C. M., Padovani, P. 1995, PASP, 107, 803

Wehrle, A. E., Cohen, M. H., Unwin, S. C., et al. 1992, ApJ, 391, 589 